\title{
DEPRESSION OF LACTASE ACTIVITY IN THE SMALL IN- TESTINE OF INFANT RABBITS BY CANDIDA ALBICANS
}

\author{
RUTH F. BISHOP AND G. L. BARNes* \\ Department of Gastroenterology, Royal Children's Hospital, Melbourne, and Department of \\ Paediatrics, University of Melbourne, Victoria, Australia
}

A SUR VEY of the microbial flora of the alimentary tract of 39 infants suffering from acute gastro-enteritis revealed Candida albicans growing abundantly in the stomach and duodenum in one-third of the patients (Bishop, Barnes and Townley, 1973). Growth in the duodenum was associated with depression of disaccharidase activity in the gut mucosa; and there was a statistically significant association of this with lactase depression (Barnes, Bishop and Townley, 1973). Invasion of duodenal mucosa by C. albicans was not seen in stained sections. These results suggested that some product of growth of $C$. albicans in the gut lumen might damage the brush border of the intestinal epithelial cell, resulting in depression of disaccharidase activity.

To test this hypothesis, strains of $C$. albicans isolated from infants with gastro-enteritis were injected into ligated loops of infant rabbit gut. This paper describes the effect of growth of $C$. albicans in vivo on lactase activity in the small intestine.

\section{MATERIALS AND METHODS}

Choice of animal. Infant albino rabbits, locally inbred, aged from 19-20 days and partly weaned, were used. The choice of this particular model was made for three reasons: (1) the normal flora of the small intestine of the rabbit resembles that of man (Smith, 1965); (2) experience with strains of Escherichia coli shows that the rabbit gut reacts better than that of other animals to human enteropathogens (Smith and Gyles, 1970; Moon and Whipp, 1971); and (3) infant rabbits still possess measurable amounts of lactase activity at weaning (Doell and Kretchmer, 1962).

Surgical procedure. The animals were fasted for 24 or 48 hours pre-operatively, depending on age, and were given water ad libitum. Anaesthesia was induced with intravenous Nembutal and maintained with ether and oxygen. The surgical procedure was identical with that described by Burrows and Musteikis (1966). Loops measuring 4-6 cm were tied along the whole small intestine with the exceptions of the 10-12 cm of duodenum and the distal $6-8 \mathrm{~cm}$ of ileum. Inoculations of $1.0-1.5 \mathrm{ml}$ of a Sabouraud-broth culture of C. albicans and of a similar volume of sterile medium were made into alternate loops. The rabbits were fasted for 20 hours post-operatively and then killed with ether. The entire small intestine was dissected. Loops were inspected for distension and then sampled for microbial culture. Specimens of intestine for histological examination were placed in Bouin fixative. Specimens for lactase assay were wrapped in Parafilm, stored at $-20^{\circ} \mathrm{C}$, and later assayed by the method of Dahlqvist (1964). Other disaccharidases were not assayed.

Microbiological methods. Ten strains of $C$. albicans, isolated from the duodenal contents of infants with acute gastro-enteritis, were inoculated into gut loops. They were maintained

Received 30 July 1973; accepted 4 Oct. 1973.

* Present address: Department of Paediatrics, University of Otago, Dunedin, New Zealand.

J. MED. MICROBIOL.-VOL. 7 (1974) 
at $4^{\circ} \mathrm{C}$ on nutrient-agar slopes, subcultured into Sabouraud broth 20 hours before testing and incubated aerobically at $37^{\circ} \mathrm{C}$. Numbers of yeast cells per $\mathrm{ml}$ were estimated by direct count in a haemocytometer. The inoculum contained approximately $10^{8}$ yeast cells per $\mathrm{ml}$ in $1.0-1.5 \mathrm{ml}$ of fluid. Each strain of C. albicans was tested in at least four rabbits at different levels of the gut.

At necropsy, specimens of peritoneal fluid and heart blood were obtained before the gut was disturbed. The contents of test and control loops were sampled by washing out with $0.5 \mathrm{ml}$ of sterile nutrient broth. Gut-lumen washings and peritoneal swabs were plated on to horse-blood agar, MacConkey agar, Sabouraud agar and Rogosa agar, all incubated aerobically at $37^{\circ} \mathrm{C}$; and horse-blood agar and Rogosa agar incubated anaerobically with $5-10 \%$ added $\mathrm{CO}_{2}$. Heart blood was incubated in liquid medium for 7 days, then sub-cultured on to the above media. The numbers of organisms present in the intestinal contents were estimated by the semi-quantitative plating technique of Cregan and Hayward (1953). Growth of yeasts was estimated from the degree of growth on Sabouraud agar, and by direct counting in a hemacytometer. No attempt was made to identify systematically all the micro-organisms present, but all colonies were identified to the generic level. A Gram-stained smear of loop contents was always examined as a check on the adequacy of the culture techniques.

Evaluation of results. Results were rejected (1) if $10^{6}$ C. albicans per $\mathrm{ml}$ was recovered from the test loop, (2) if the indigenous flora was markedly different qualitatively or quantitatively in test and control loops, (3) if a known intestinal pathogen formed part of the indigenous flora, e.g., Clostridium welchii, (4) or if at necropsy there was reason to suspect that ligation had caused infarction. In addition, results were rejected if (5) rabbits showed consistently low levels of lactase activity in control loops $(<0.5$ units of activity per $g$ wet weight of tissue). It was assumed that the physiological fall in lactase shortly after weaning (Doell and Kretchmer, 1962) had already occurred in these animals.

Statistical analysis. Results from matched pairs of loops were analysed by means of a one-tailed Student distribution. Test and control loops were matched for indigenous flora and level of intestine in the same animal.

\section{RESULTS}

Thirty-nine test loops that received $C$. albicans and 34 control loops that received sterile broth satisfied the criteria listed above, and there was a slight but consistant decrease in lactase activity in the test loops when compared with control loops (fig. 1).

The results from 25 matched pairs of these loops were analysed statistically (fig. 2). Mean lactase activities per $g$ wet weight of tissue were 1.58 for $C$. albicans loops, and 1.83 for control loops; standard error of the difference was $0 \cdot 11$. This shows a statistically significant decrease of lactase activity in loops inoculated with $C$. albicans $(t=2.10 ; 0.025>\mathrm{P}>0.01)$.

A consistent macroscopic effect of the growth of $C$. albicans on the lumenal surface of the loops was not observed, and none of the loops was distended. During the 20 hours of incubation in the gut lumen, the yeast form of $C$. albicans inoculated was partly converted into the mycelial form. Histological study by means of the light microscope showed normal epithelium in the majority of loops. There was no evidence of mucosal invasion by $C$. albicans in its yeast or mycelial form.

\section{Discussion}

Candida albicans is rarely listed as an enteric pathogen in reviews on the aetiology of infantile diarrhoea. In a careful study of enteric candidiasis, 


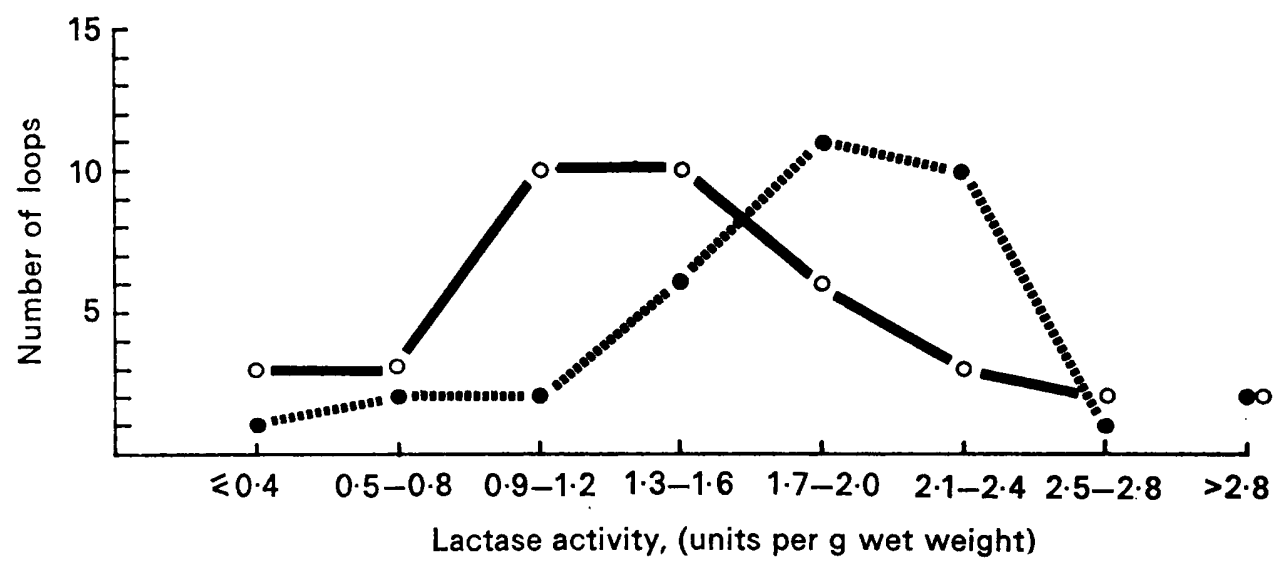

FIG. 1.-Amount of lactase activity in ligated loops of small intestine of infant rabbits: $0=$ Candida albicans inoculated into loops; $\bullet=$ sterile broth inoculated into loops.

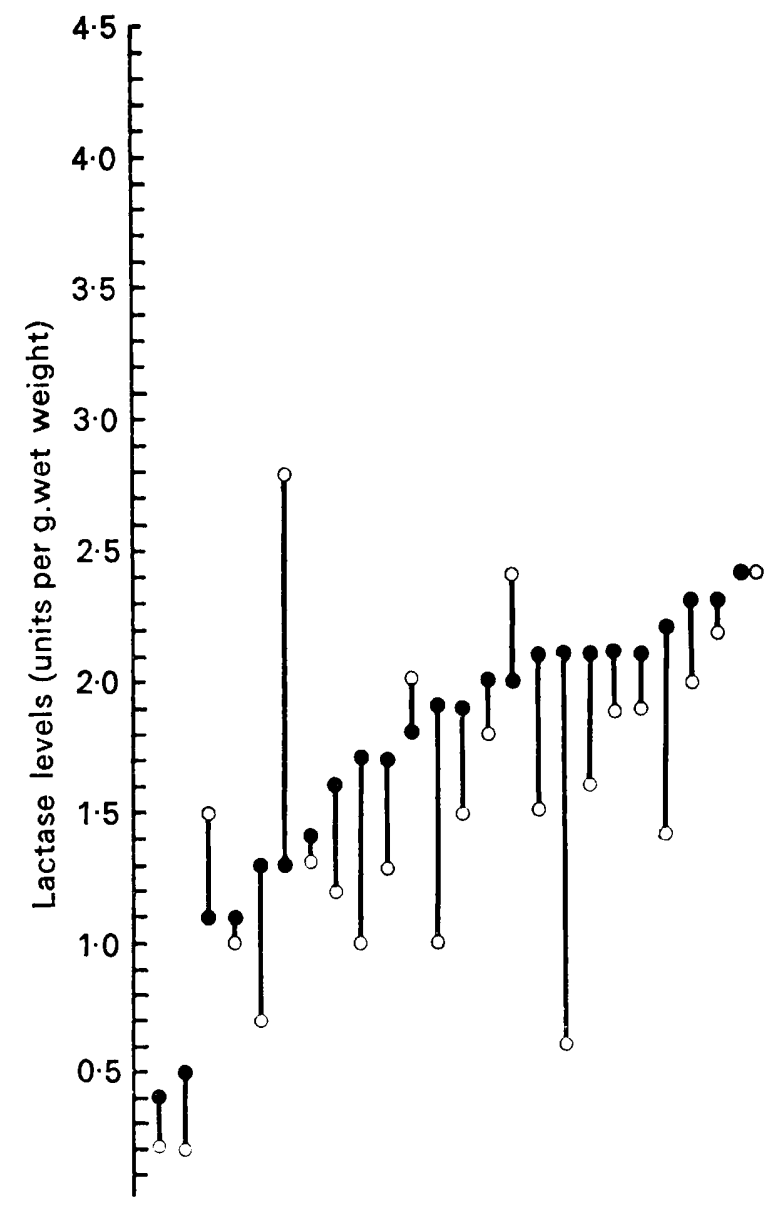

FIG. 2.-Amount of lactase activity in matched pairs of rabbit small bowel loops: $0=$ Candida albicans inoculated into loops; $\bullet=$ sterile broth inoculated into loops. 
Kozinn and Taschdjian (1962) comment that the disease is seldom suspected and even less commonly diagnosed ante-mortem. Their observations clearly establish C. albicans as an important cause of diarrhoea, often of prolonged duration, in infants and children, and are supported by the findings of Rogers (1966). The infection may be primary or may supervene after an initial infection by other intestinal pathogens; and it can be present without signs of oral thrush. The diarrhoea responds well to oral Nystatin.

In a recent survey of acute gastro-enteritis we isolated C. albicans from the stomach and duodenum of one-third of the infants examined (Bishop et al., 1973). Growth in the duodenum was associated with depression of lactase activity (Barnes et al., 1973). It was not clear whether the lactase depression was a primary result of the growth of $C$. albicans, whether it was caused by an unknown agent (presumably viral), or whether unabsorbed disaccharides in the gut lumen stimulated the overgrowth of the yeast.

Our results show that $C$. albicans primarily depresses lactase activity in vivo in the infant rabbit. There was no evidence of mycelial invasion by the yeast; hence this yeast must have the ability to damage the intestinal epithelial cell by its growth in the gut lumen. The mechanism by which it produces such damage is open to conjecture. It is obvious from our results that $C$. albicans does not produce an enterotoxin, similar to that of $E$. coli, that causes distension of ligated loops, and it is unlikely that $C$. albicans produces potent soluble exotoxins (Winner and Hurley, 1962). However, there is evidence of endotoxinlike products of growth; Iwata and Uchida (1969) extracted such a substance that caused oedema and congestion of the small intestine when injected into the peritoneal cavity of mice; histologically there was cellular infiltration in the submucosa and lamina propria.

C. albicans can probably also affect lactase activity in the small bowel of the human infant. Even if it is not responsible for the initial damage to the small bowel in infantile gastro-enteritis, rapid colonisation of the gut by this yeast might result in prolonged lactase depression.

This has interesting implications for the treatment of enteritis in infancy and its common sequel-disaccharide intolerance. The use of anti-fungal agents might reduce dietary problems in the convalescent stage in some infants with acute infectious diarrhoea.

\section{SUMMARY}

Strains of Candida albicans isolated from the duodenum of infants with acute gastro-enteritis were inoculated into ligated segments of small intestine of infant rabbits. Twenty hours later, the lactase activity in the mucosa of these segments was compared with the lactase activity in similar segments into which sterile broth had been inoculated. Analysis of the results from 25 matched pairs of loops showed a statistically significant decrease in lactase activity in the loops that had received C. albicans. We suggest that the depression of lactase activity observed in infants with gastro-enteritis may be caused or perpetuated by the growth of $C$. albicans in the duodenum. 
We gratefully acknowledge the technical assistance of Mr Max Murray, Mrs Rosa Kaye and Mr George Cauchi. R. F. B. was in receipt of a Royal Children's Hospital Research Foundation grant.

\section{REFERENCES}

Barnes, G. L., Bishop, R. F. AND Townley, R. R. W. 1973. Microbial flora and disaccharidase depression in infantile gastroenteritis. Unpublished observations.

Bishop, R. F., BARNes, G. L. AND TOWNLEY, R. R. W. 1973. Microbial flora of stomach and small intestine in infantile gastroenteritis. Unpublished observations.

BurRows, W. AND Musterkis, G. M. 1966. Cholera infection and toxin in the rabbit ileal loop. J. infect. Dis., 116, 183.

Cregan, J. AND Hayward, N. J. 1953. The bacterial content of the healthy human small intestine. Br. med. J., 1, 1356.

DAHLQvist, A. 1964. Method for assay of intestinal disaccharidases. Analyt. Biochem., $7,18$.

Doell, R. G. AND Kretchmer, N. 1962. Studies of small intestine during development. I. Distribution and activity of $\beta$-galactosidase. Biochem. biophys. Acts, 62, 353.

IwATA, K. AND UCHIDA, F. 1969. Canditoxin, a new toxic substance from a strain of Candida albicans. Antonie van Leeuwenhoek, 35, Suppl. E39.

Kozinn, P. J. AND Taschdjian, C. L. 1962. Enteric candidiasis. Diagnosis and clinical considerations. Pediatrics, Springfield, 30, 71.

Moon, H. W. AND WHIPP, S. C. 1971. Systems for testing the enteropathogenicity of Escherichia coli. Ann. N.Y. Acad. Sci., 176, 197.

ROGERS, K. B. 1966. Candida infections in paediatrics. In Symposium on candida infections, edited by H. I. Winner and R. Hurley, London, p. 179.

SмIтH, H. W. 1965. The development of the flora of the alimentary tract in young animals. J. Path. Bact., 90, 495.

SMITH, H. W. AND Gyles, C. L. 1970. The effect of cell-free fluids prepared from cultures of human and animal enteropathogenic strains of Escherichia coli on ligated intestinal segments of rabbits and pigs. J. med. Microbiol., 3, 403.

WINNER, H. I. AND HURLeY, R. 1964. Candida albicans, London, p. 212. 